\title{
COMPARISON OF THE DECAY HEAT REMOVAL SYSTEMS IN THE KALIMER-600 AND DSFR
}

\author{
KWI-SEOK HA* and HAE-YONG JEONG \\ Korea Atomic Energy Research Institutes \\ 1045 Daedeok-daero, Yuseong-gu, Daejeon, Korea \\ *Corresponding author. E-mail : ksha@kaeri.re.kr \\ Received April 01, 2011 \\ Accepted for Publication August 09, 2011
}

A sodium-cooled demonstration fast reactor with the KALIMER-600 as a reference plant is under design by KAERI. The safety grade decay heat removal system (DHRS), which is important to mitigate design basis accidents, was changed in the reactor design. A loss of heat sink and a vessel leak in design basis accidents were simulated using the MARS-LMR system transient analysis code on two plant systems. In the analyses, the DHRS of KALIMER-600 had a weakness due to elevation of the overflow path for the DHRS operation, while it was proved that the DHRS of the demonstration reactor had superior heat transfer characteristics due to the simplified heat transfer mechanism.

KEYWORDS : Sodium-cooled Fast Reactor, Decay Heat Removal System, MARS-LMR, Loss-of-Heat-Sink, Vessel Leak

\section{INTRODUCTION}

KAERI (Korea Atomic Energy Research Institute) developed KALIMER (Korea Advanced LIquid MEtal Reactor)-600[1] as a reference fast reactor design of Korea, completing the work in 2009. The KALIMER-600 is a pool type sodium cooled fast reactor of which the electric power is $600 \mathrm{MWe}$ and the core is loaded with metallic fuel. The heat transport system of the design consists of a Primary Heat Transport System (PHTS), Intermediate Heat Transport System (IHTS), Steam Generating System (SGS), and Decay Heat Removal System (DHRS).

During normal operation, the sodium coolant in the PHTS transfers heat from the reactor core to the sodium in the IHTS through the Intermediate Heat Exchangers (IHXs), and the sodium in the IHTS transports heat to the once-through type steam generator with helical tubes. At the steam generator, the subcooled water is converted into superheated steam using the transported heat. Figure 1 shows the configuration of the heat transport system of KALIMER-600.

One of the most important designs of a nuclear reactor is the DHRS for the safe and reliable decay heat removal. During a post shutdown the decay heat is removed through the DHRS, which is connected to the steam generators; however, the system cannot be guaranteed in an accident situation if it is not designed as a safety grade level. The safety system has to be designed for the prevention of faults and accident sequences with the potential to lead to an unacceptable radioactive release. This special DHRS improves the reliability of a plant. The DHRS of the KALIMER-600 conceptual design is composed of three diverse systems to enhance the plant safety and reliability: a non-safety grade steam/feedwater system, a non-safety grade Intermediate Reactor Auxiliary Cooling System (IRACS), and a safety grade PDRC (Passive Decay heat Removal Circuit).

The PDRC is used to remove heat directly from the coolant in the reactor vessel through two independent loops. Each loop is composed of a sodium to sodium decay heat exchanger (DHX), which is supported by the reactor roof, a sodium to air heat exchanger (AHX) as the ultimate heat sink, a sodium expansion tank, and an intermediate sodium pipe connecting the DHX and AHX. The DHX, located inside the DHX support barrel, is partially submerged in the cold pool sodium to prevent sodium solidification in the intermediate loop of the PDRC and to minimize heat loss to the environmental atmosphere under normal operation modes [2].

Under accident conditions, the level of the cold pool rises and the DHX is fully immersed, because the PHTS pump stops operating following the reactor shutdown. In addition, the hot pool sodium expands, because the decay power from the core is greater than the heat removed by normal heat transport systems. Finally, the hot pool sodium overflows into the DHX support barrel. The decay heat is then transferred to the secondary sodium by global natural circulation of the PHTS sodium, the path of which is composed of the core, hot pool, shell side of the DHX, cold pool, and core inlet plenum. 


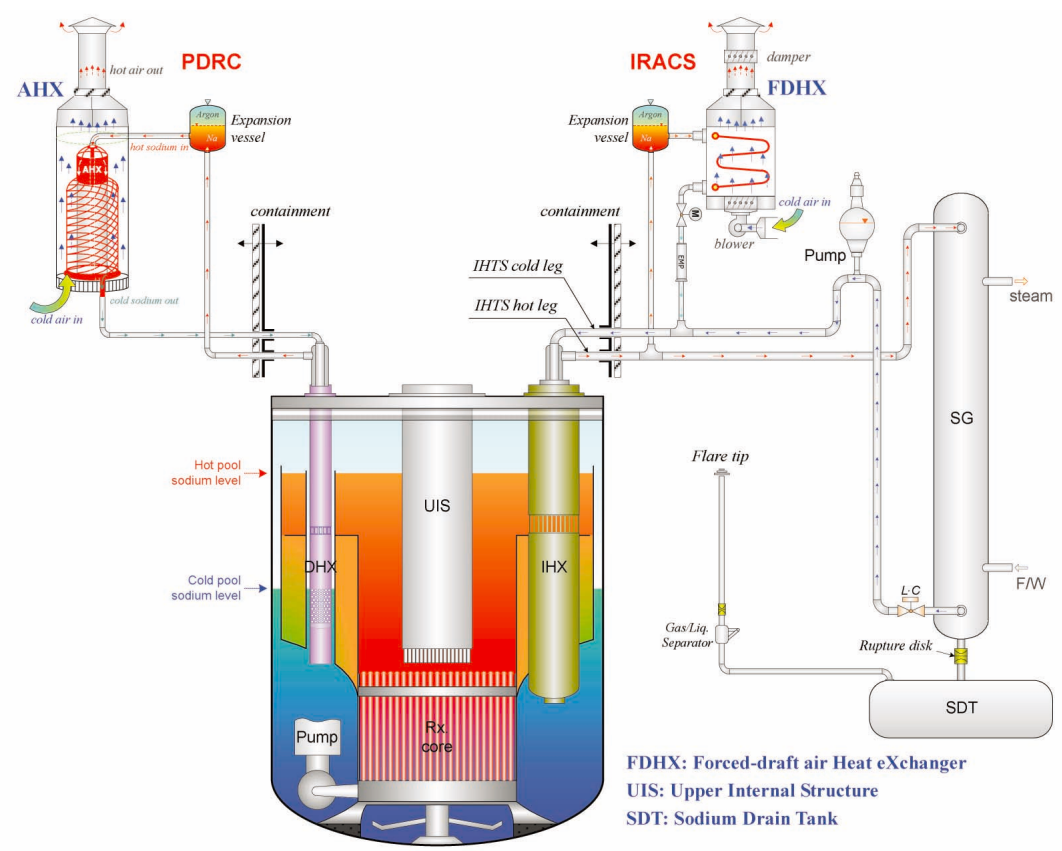

Fig. 1. Configuration of the Heat Transport System for KALIMER-600

In general, the location of the DHX plays an important role in the determination of the AHX size, because the temperature difference of the hot and cold pools is about $150{ }^{\circ} \mathrm{C}$ in a fast reactor. Thus, the AHX size based on the cold pool temperature has to be much larger than that based on the hot pool temperature in order to remove the same capacity of heat. Thus, it is of great advantage economically to cool the hot pool sodium, as in the DHRS of the European Fast Reactor [3]. The operation mechanism of the DHRS of KALIMER-600 is similar to that of cooling hot pool sodium due to the use of the flooded sodium from the hot pool, although the DHX is in the cold pool sodium.

The other advantage of the DHRS of KALIMER-600 is a fully passive concept without any active operating equipment; in particular, an emergency diesel generator is not needed. However, the DHRS is not available in accidents where the sodium level in the cold pool is below the DHX, due to the running of PHTS pumps, or where overflow from the hot pool to the cold pool does not occur, as the sodium level of the hot pool is lower than the elevation of the path for the overflow of a reactor barrel. Thus, the design concept of the DHRS of KALIER-600 should be replaced with a concept that would be available in all postulated accidents.

Recently, KAERI designed a Demonstration Sodiumcooled Fast Reactor (DSFR) to demonstrate KALIMER600. However, a new design concept for the DHRS of the DSFR was suggested owing to the aforementioned disadvantages. In this paper, the thermal hydraulic behaviors of the DHRS of KALIMER-600 for two postulated events are compared with the behaviors of the new DHRS of the DSFR to clarify the reasons for changing the DHRS of KALIMER-600.

\section{DESCRIPTION OF THE DHRS OF KALIMER-600 AND THE DSFR}

The PDRC of KALIMER-600 is comprised of two independent loops owing to its basis on a full passive concept. Each loop can remove $50 \%$ of the core decay heat in half an hour after a reactor scram. During pump operation, as shown in figure 2(a), $30 \%$ of the effective heat transfer tube length of the DHX is immersed into the cold pool sodium to maintain a minimum loop sodium flow with minimized heat loss into the environment. A small amount of sodium in the cold pool travelling up the gap between the DHX support barrel and the DHX shroud flows into the DHX shell side through the perforations of the DHX shroud. The sodium transfers its heat to the tube side sodium. The naturally circulated sodium flow is formed by the density difference between the hot sodium of the DHX tube side and the cold sodium of the AHX tube side cooled by the density driven air flow.

In design basis events, the sodium level of the cold pool rises and the DHX is fully submerged in the cold pool sodium owing to the increase of the cold pool level due to a PHTS pump trip after a reactor shutdown, as depicted in Figure 2(b). In this mode, the heat transfer rate is slightly increased relative to the normal operation mode due to the extension of the effective heat transfer 


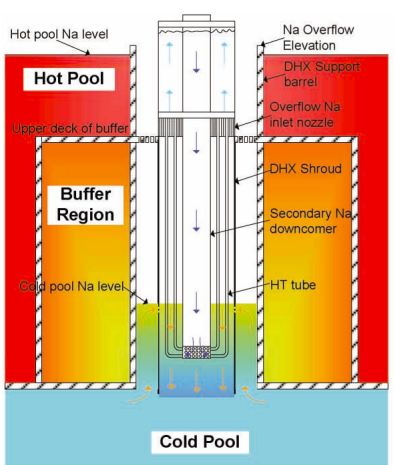

(a) Normal operation

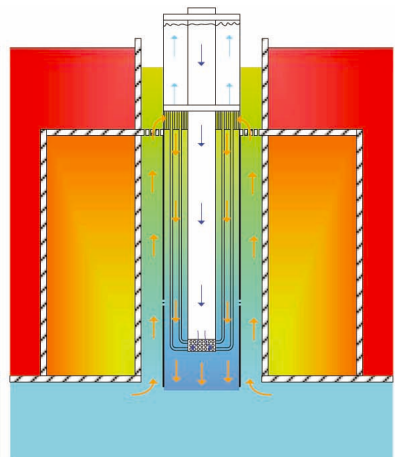

(b) Before Overflow

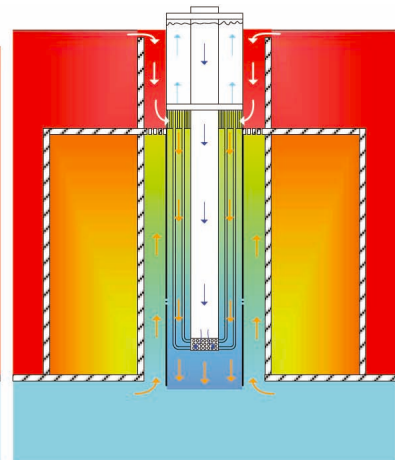

(C) Overflow

Fig. 2. Operation Modes of the DHX of KALIMER-600

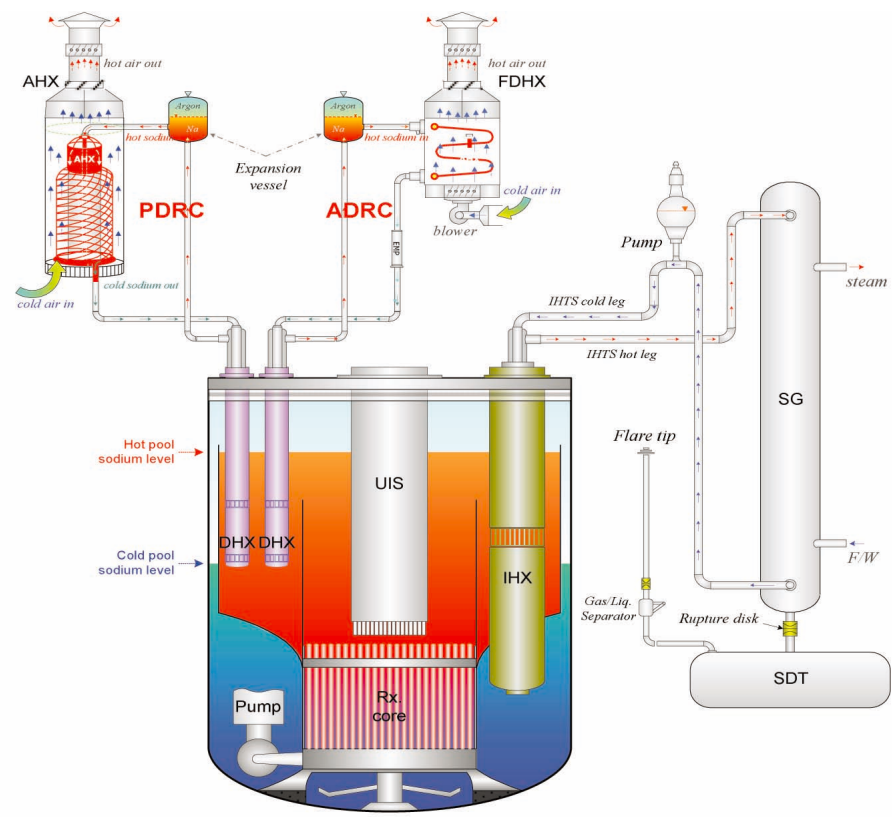

Fig. 3. Configuration of the Heat Transport System for DSFR

length. However, the mismatch between the decay power and the heat removal by the DHRS because of the unavailability of the normal heat transport path causes the hot pool level to increase.

The hot pool sodium flows over the DHX barrel if the sodium level rises higher than the elevation of the sodium overflow path. It is then mixed with the sodium inside the DHX barrel, and the coolant continuously flows into the shell-side of DHX, as shown in Figure 2(c). The PDRC is a totally self-regulating system, since the heat removal capacity of the PDRC is directly proportional to the pool sodium temperature variation as long as the overflow is maintained. Thus, the removal of decay heat is effectively accomplished using a purely passive concept depending on density-driven flow without any operator action or any active component actuation. However, the PHTS pumps should be tripped in all accidents to immerse the DHX, which acts against Unprotected Transient of Over Power (UTOP). Also, the operation mechanism of the DHRS of KALIMER is very complex and the heat removal is limited by the overflow, because the DHRS was designed based on the sodium temperature of the hot pool, whereas the DHX is put in the cold pool.

In the conceptual design of the DSFR, the DHX is fully submerged in the sodium of the hot pool annular region (the buffer region in KALIMER-600) to remedy some of the drawbacks of the DHRS of KALIMER-600, as shown in Figure 3. For buildup of the natural circulation head under accident situations, the DHX is located in the top part of the annular region and the IHX is set in the 
Table 1. Comparison of Design Parameters between KALIMER-600 and DSFR

\begin{tabular}{|c|c|c|}
\hline Parameter & KALIMER-600 & DSFR \\
\hline Power, MWt & 1523.4 & 1546.4 \\
\hline Safety Decay Heat Removal & 2-Loop PDRC & 2-Loop PDRC, 2-Loop ADRC \\
\hline Core I/O Temp. $\left({ }^{\circ} \mathrm{C}\right)$ & $390.0 / 545.0$ & $365.0 / 510.0$ \\
\hline PHTS Flow Rate(kg/s) & 7731.3 & 8366.1 \\
\hline No. of PHTS Pumps/IHXs & $2 / 4$ & $2 / 4$ \\
\hline IHX I/O Temp. $\left({ }^{\circ} \mathrm{C}\right)$ & $320 / 526$ & $305.4 / 502.0$ \\
\hline IHTS Flow Rate(kg/s) & 5800.7 & 6146.0 \\
\hline Steam Flow Rate(kg/s) & 663.25 & 689.3 \\
\hline Steam Temperature $\left({ }^{\circ} \mathrm{C}\right)$ & 503.1 & 471.2 \\
\hline Steam Pressure(MPa) & 16.5 & 17.3 \\
\hline Heat Removal Rate/Each DRC, MW & 8.25 & 9.0 \\
\hline
\end{tabular}

lower part. Thus, the hot pool is broadened to the buffer region of KALIMER-600 and the upper deck of the buffer region is removed. On the other hand, a damper should be installed at the AHX to cut short heat loss to the environmental atmosphere.

The disadvantages of the DHRS of KALIMER are eliminated in the DSFR, while the passivity of the DHRS of DSFR is slightly reduced due to the dampers, pumps, and air blowers, which are operated by an emergency diesel generator. The difference of the PDRCs of KALIMER600 and DSFR lies in the inclusion of a damper in the latter. The damper of the DSFR is needed to prevent heat loss to the environment during normal operation and is designed with a fail-open concept.

The heat transfer mechanism in the DHRS, which does not include the overflow concept in the DSFR, is simple compared with that in KALIMER-600. In a postulated accident, generally, feedwater to the SG is not supplied owing to the assumption of the loss of offsite power. Thus, the heat removal by the SG is reduced, while the heat removal by the DHRS is continuously increased if the AHX damper is open.

\section{INITIAL CONDITIONS AND CALCULATION MODELS FOR ACCIDENT ANALYSES}

The KALIMER-600 core has a breakeven breeding characteristic, consisting of 1 ultimate shutdown assembly, 117 inner fuel assemblies, 96 middle fuel assemblies, 120 outer fuel assemblies, and 369 non-fuel assemblies. The core inlet temperature is $390.0^{\circ} \mathrm{C}$ and the core outlet temperature is $545.0^{\circ} \mathrm{C}$. The height and diameter of the reactor vessel are $11.5 \mathrm{~m}$ and $18.5 \mathrm{~m}$, respectively, and the active core height is $94 \mathrm{~cm}$. Table 1 compares the overall design parameters for KALIMER-600 and the DSFR.

The thermal power of the DSFR is $1548.2 \mathrm{MW}$ and there are 150 inner fuel assemblies, 174 outer fuel assemblies, and 349 non-fuel assemblies. The core inlet/ outlet temperatures are reduced to $365.0 / 510.0{ }^{\circ} \mathrm{C}$ because of the clad material change from Modified HT-9 to HT-9. The height and diameter of the vessel are 11.9 $\mathrm{m}$ and $16.5 \mathrm{~m}$, and the active core height is $89 \mathrm{~cm}$. The DHRS of the DSFR is composed of 2 loops of the PDRC and 2 loops of the ADRC (Active Decay Heat Removal Circuit), where the heat removal capacity of each loop is $9 \mathrm{MW}$ while that of KALIMER-600 is 8.25 MW. An electro-magnetic pump and an air blower are equipped in each active loop.

To investigate the effectiveness of each DHRS in two plant systems, two postulated accidents are analyzed using the MARS-LMR (Multi-dimensional Analysis of Reactor Safety-Liquid Metal Reactor) code [4]. A sodium coolant property table, wall heat transfer coefficients, and the friction factor correlations related to a liquid metal reactor system were added to the MARS code [5] for use in an analysis of transients for a liquid metal cooled reactor system. An accident of Loss Of Heat Sink (LOHS) was adopted to assess the long term cooling effect by the safety grade DHRS and a reactor vessel leak accident was selected to investigate the availability of the DHRS in the event of a reduced sodium level of the hot pool. Figure 4 shows the MARS-LMR calculation nodalizations for two plant systems.

A large change in nodalizations is derived from the modeling of the DHX, which is represented within the gray circles. The DHX of the DSFR is located at the path 


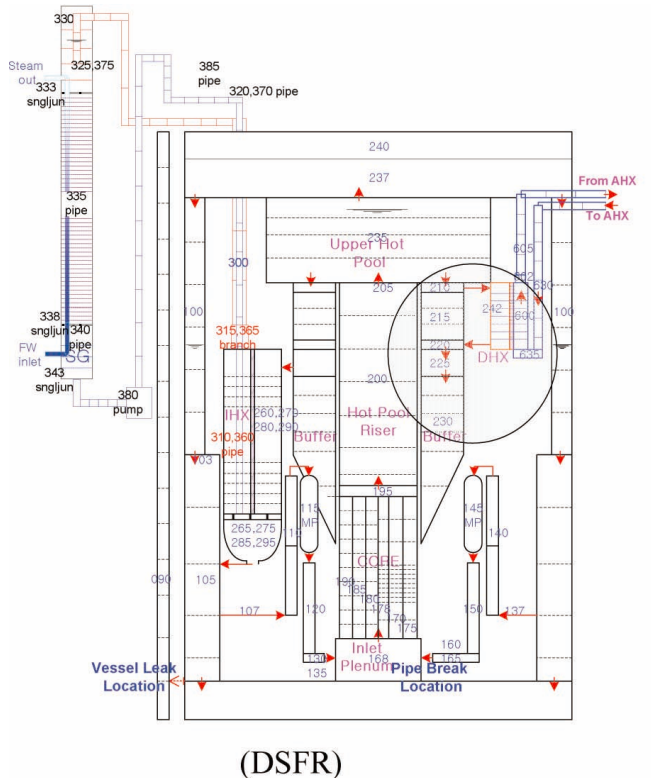

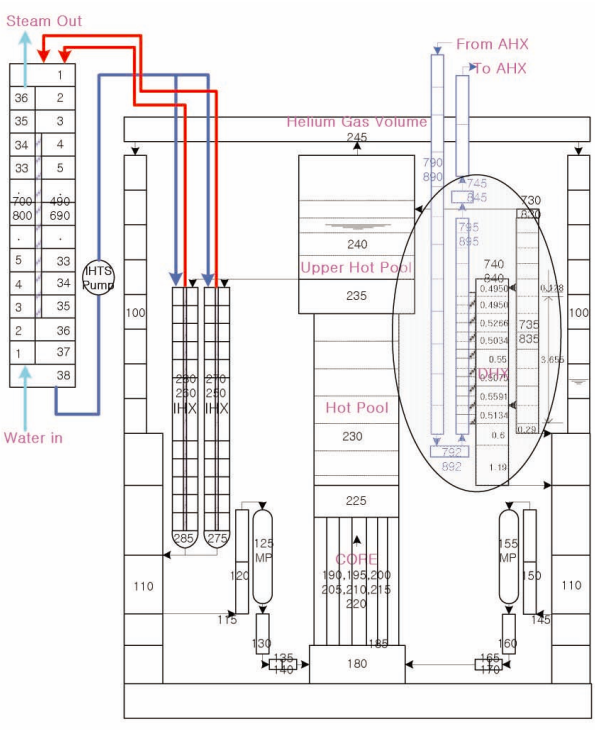

(KALIMER-600)

Fig. 4. MARS-LMR Nodalizations for DSFR and KALIMER-600

of the down flow in the peripheral region of the hot pool. Thus, the main flow reaches the IHX inlet via the annular region of the hot pool, while a small amount of sodium flows in the DHX shell side parallel to the main flow. The DHX is located above the IHX to enlarge the natural circulation head under accident situations. On the other hand, the DHX of KALIMER-600 is placed in the cold pool. The barrel, in which the DHX shroud is contained, is connected with the upper hot pool node, and the upper shell side of the DHX is linked by the middle node of the barrel. The cold sodium cooled by the DHX is dumped into the cold pool through the DHX outlet junction, and the bottom part of the DHX barrel is also connected with the cold pool to simulate the cooling mode, as shown in Figures 2(a) and (b).

In KALIMER-600, the buffer region is not modeled, because sodium in the region is nearly stagnated; however, in the DSFR, the region where sodium flows down contains 4 IHXs and 4 DHXs. The IHX outlet is connected with the second junction of the cold pool component. The sodium of the main flow is pumped to the inlet plenum from the third junction of the cold pool by the pump node, and is heated at the core region, which is divided into seven parallel channels. The hot sodium travels up through the hot pool center region.

\section{COMPARISON OF THE DHRS PERFORMANCE IN POSTULATED ACCIDENTS}

\subsection{Analysis of a Loss of Heat Sink}

A LOHS accident is initiated by a loss of feedwater

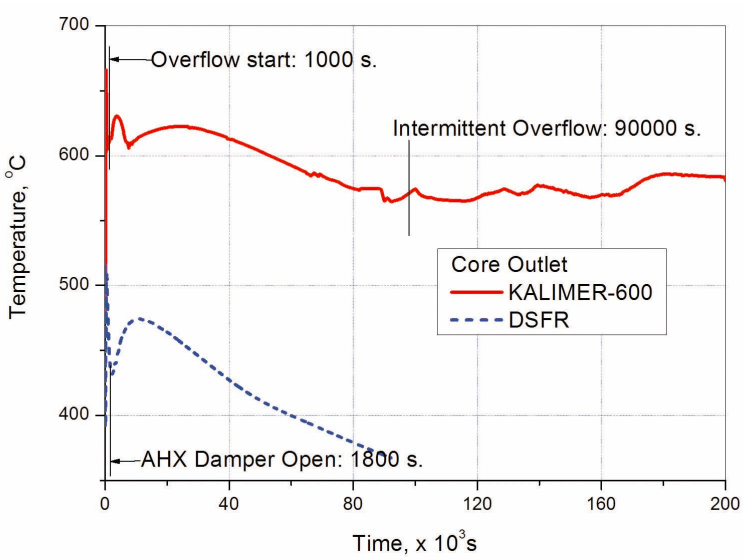

Fig. 5. Core Outlet Temperatures

supply to all steam generators. It was assumed that a loss of offsite power occurred at 5 seconds after the reactor scram as an aggravating failure of the accident. Figure 5 compares the core outlet temperatures of KALIMER-600 and the DSFR for this transient. The behaviors of the early phase in the calculations showed a large difference according to the initial conditions; however, the trends of the later phase were dominated by the heat removal of the DHRS of the two plant systems. The decay heat produced in the reactor core was very similar in the two systems due to having almost the same initial power. The reactor was scrammed by the signal of the high core outlet temperature, which caused a rapid drop in the temperature. All pumps in the PHTS and IHTS were tripped and experienced a coastdown; the temperature 
was then raised until the heat removal by the DHRS exceeded the core decay power.

In KALIMER-600, an overflow in which the hot pool sodium flooded into the DHX to activate the heat removal by the PDRC started at around 1,000 seconds by the sodium expansion. The flow was increased and sufficiently formed to operate according to its design purpose at around 10,000 seconds, as shown in Figure 6. A momentary temperature drop occurred at that time because the inflow into the core of the cold sodium accumulated at the cold pool below the DHX. The core decay power was still higher than the heat removal, as shown in Figure 7, and consequently the core outlet temperature continued to increase. The temperature finally decreased, because the heat removal surpassed the core power from about 29,000 seconds.

As the decay power was reduced and the sodium in the PHTS was cooled, the coolant level in the hot pool descended, which decreased the overflow rate. At around 90,000 seconds, the level reached the overflow elevation, and the decay heat was then removed by the intermittent overflow, as the modes of (c) and (b) in Figure 2 were

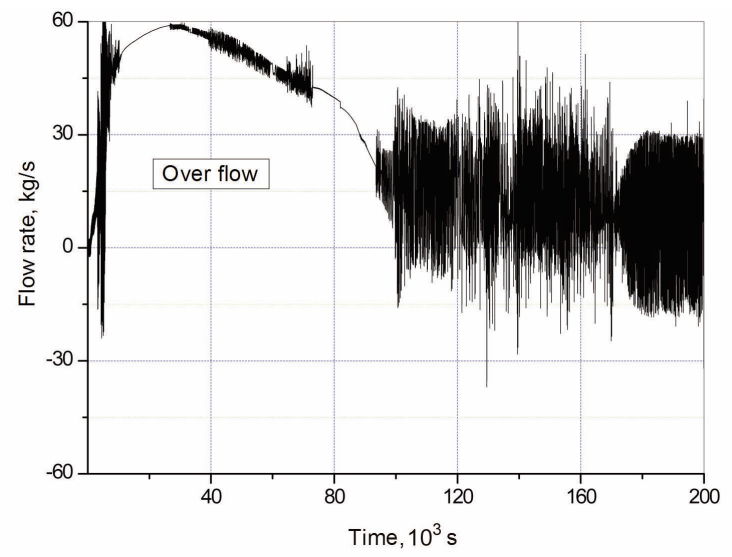

Fig. 6. Overflow Rate of KALIMER-600

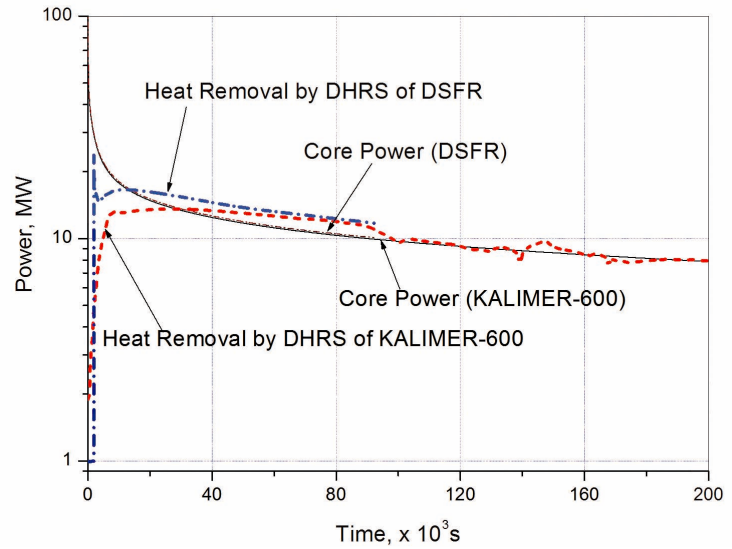

Fig. 7. Core Power vs. Heat Removal by DHRS repeated, because the cooling capacity by (b) mode was not enough. This means that the core outlet temperature is determined by the overflow elevation. The temperature was maintained as $575^{\circ} \mathrm{C}$, higher than that $\left(545^{\circ} \mathrm{C}\right)$ at the $100 \%$ power condition, because of the higher elevation of the overflow path relative to the normal operation level. The temperature satisfies the safety criterion of $600{ }^{\circ} \mathrm{C}$ for the core outlet; however, too much time is required to switch the plant to safe mode (hot or cold shutdown) after the accident.

The PDRC of KALIMER-600 is passively operated without any operation of active equipment whereas the AHX damper of DSFR is opened by an emergency power supply. In the accident analysis of the DSFR, it is conservatively assumed that two loops of the DHRS are available and the damper is opened 30 minutes after the accident initiation by an operator action. The core outlet temperature in the initial phase of the accident was calculated to be about $150{ }^{\circ} \mathrm{C}$ less than the case of KALIMER-600 due to the difference of the heat removal in the steam generators. However, decay power larger than the heat removal led to a rise in the core outlet temperature. The temperature reached a peak of $475{ }^{\circ} \mathrm{C}$ at around 12,000 seconds, and thereafter continued to decrease through greater heat removal than the decay power. In the DSFR, the DHX is always submerged in the hot pool sodium. As a result, only the hot sodium passes through the DHX shell side, while in the DHX inlet of KALIMER-600, the overflowed hot sodium is mixed with the cold sodium moving up from the cold pool through the inter-space between the DHX support barrel and the shroud. At 60,000 seconds after the beginning of the accident, the temperature reached $400{ }^{\circ} \mathrm{C}$, and the plant could be maintained in a hot shutdown state. From this analysis it is concluded that the DHRS of the DSFR is much more effective than the system of KALIMER-600.

\subsection{Analysis of a Reactor Vessel Leak}

A sound design and periodic in-service inspections for a reactor vessel wall ensure that only a small size $\left(\sim 10^{-6} \mathrm{~m}^{2}\right)$ leak can occur within the design basis; however, as a hypothetical design basis accident such as a large break loss of coolant accident (LBLOCA) in a water reactor, a rupture of $0.001 \mathrm{~m}^{2}$ in the bottom of a reactor vessel was considered. The leak location is shown in the bottom left part of the DSFR nodalization of Figure 4. There is an annular space between the exterior of the reactor vessel and the guard vessel, and the leaked sodium is confined in the annular space.

It is important to ensure that the topmost component in the sodium pool is not left uncovered due to this accident. In the KALIMER-600 design, the IHX inlet windows are located at $2.9 \mathrm{~m}$ below the normal hot pool level and the overflow paths are $0.3 \mathrm{~m}$ higher than the normal level. As mentioned in section IV.1, the DHRS of 
KALIMER-600 is operated as intended when the overflow is formed. As shown in Figure 8, the hot pool level was decreased by $0.85 \mathrm{~m}$, because the sodium in the PHTS was discharged into the inter-space until 3,500 seconds. A reactor scram occurred at 70 seconds by the signal of the low pool level. The loss of feedwater to the steam generator and all pumps trips were also triggered 5 seconds after the reactor scram according to the aggravated assumption of the loss of offsite power.

The reduced sodium level again rose due to the sodium expansion in the PHTS, which was invoked by the deterioration of the PDRC heat removal. However, the sodium level was not sufficient to force an overflow. The lack of heat removal of the PDRC caused the clad temperature to violate the safety limit of $690{ }^{\circ} \mathrm{C}$ at about 17,500 seconds, as shown in Figure 9. Thus, the DHRS of KALIMER-600 did not play a role in this accident.

On the other hand, in the calculation for the hypothetical accident in the DSFR, the hot pool sodium level was reduced by $0.67 \mathrm{~m}$, but the level was $1.57 \mathrm{~m}$ above the DHX inlet windows, and thus the DHX was always immersed in the hot pool. Thus, the heat transfer from

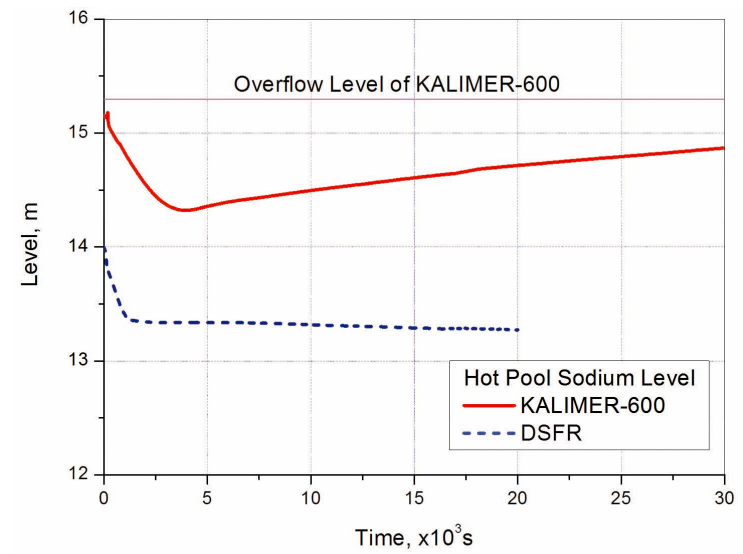

Fig. 8. Hot Pool Sodium Levels

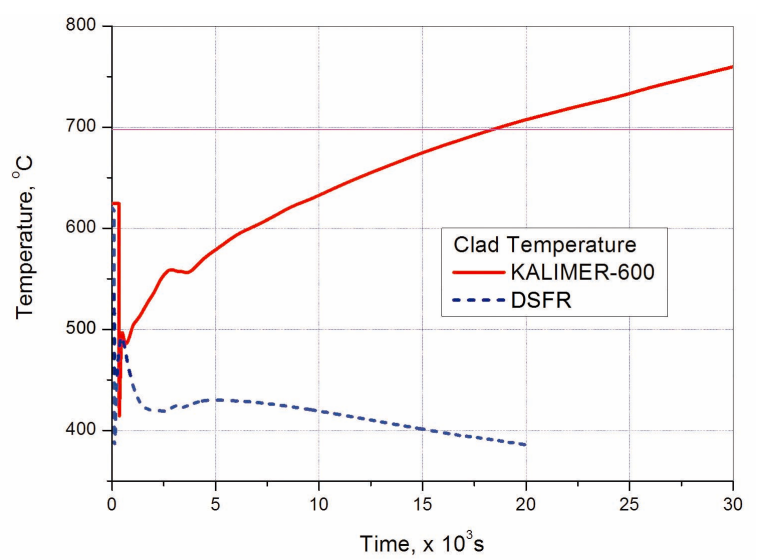

Fig. 9. Clad Temperature Behaviors the hot pool sodium to the sodium in the DHX tube worked well as designed, and the clad temperature was well below the safe shutdown condition.

\section{CONCLUSIONS}

Recently, a DSFR, the reference plant of which is the KALIMER-600, has been under design at KAERI. The overall design is almost the same as that of KALIMER600 , but the DHRS of the DSFR is greatly changed. The altered DHRS is composed of two loops of passive circuits and two loops of active circuits, and it has a superior heat transfer characteristics due to the simplified heat transfer mechanism as compared with the DHRS of KALIMER600. However, its passivity is slightly reduced due to the active components, such as the AHX damper and air blowers.

Two design basis accidents were simulated using the MARS-LMR system transient analysis code for assessing the capability of the DHRS of the DSFR in comparison with that of KALIMER-600. In the accident analyses for the loss of heat sink of KALIMER-600, the core outlet temperature was maintained at $575{ }^{\circ} \mathrm{C}$, which is higher than the rated temperature due to the elevation of the overflow path. Although it satisfies the safety criterion, this temperature may cause problems in the long term cooling of the plant under accident conditions. Furthermore, the DHRS of KALIMER-600 cannot be operated as designed during a vessel leak accident, because the overflow does not occur due to the reduced level of the hot pool. However, the DHRS of the DSFR operates well and all calculated results were proven to satisfy the safety criteria and safe shutdown conditions in long term cooling. Conclusively, the analyses showed that alteration of the DHRS is very feasible. In the future, the uncertainty in the flow split to IHX and DHX in the hot pool region will be validated experimentally.

\section{ACKNOWLEDGEMENTS}

This study was performed under the long-term nuclear research and development program sponsored by the Ministry of Education, Science and Technology of Republic of Korea.

\section{REFERENCES}

[ 1 ] Dohee Hahn, et al, "KALIMER-600 Conceptual Design Report," KAERI/TR-3381/2007, Korea Atomic Energy Research Institute, Daejeon, Korea (2007)

[2 ] Jae-Hyuk Eoh, et al, "New Design Options Free from a Potential Sodium Freezing Issue for a Passive DHR System of KALIMER,” Nuclear Technology, Vol. 170, pp. 290305 (2010)

[3 ] B. Farrar, et al, "Fast Reactor Decay Heat Removal: Approach to the Safety System Design in Japan and Europe," Nuclear Engineering and Design, Vol. 193, pp. 45-54 (1999) 
[4] Kwi-Seok Ha, et al, "Simulation of the EBR-II Loss-ofFlow Tests using the MARS code," Nuclear Technology, Vol. 169, pp. 134-142 (2010)
[ 5 ] Jae-Joon Jeong, et al, "Development of Multi-dimensional Thermal-hydraulic System Code, MARS 1.3.1," Annals of Nuclear Energy, Vol. 26, 1611 (1999) 